\title{
Hybrid Feature Fusion and Machine Learning Approaches for Melanoma Skin Cancer Detection
}

\author{
Md. Mahbubur Rahman ${ }^{1,2}$, Mostofa Kamal Nasir ${ }^{1}$, Md. Nur-A-Alam ${ }^{1,2}$,Md. Saikat Islam Khan ${ }^{1,2}$ \\ Shahab S.Band ${ }^{3 *}$, Abdollah Dehzangi ${ }^{4,5}$, Amin Beheshti ${ }^{6}$, Hamid Alinejad-Rokny $7,8,9$
}

\begin{abstract}
Skin cancer is an exquisite disease globally nowadays. Because of the poor contrast and apparent resemblance between skin and lesions, automatic identification of skin cancer is complicated. The rate of human death can be massively reduced if melanoma skin cancer can be detected quickly using dermoscopy images. In this research, an anisotropic diffusion filtering method is used on dermoscopy images to remove multiplicative speckle noise and the fast-bounding box (FBB) method is applied to segment the skin cancer region. Furthermore, the paper consists of two feature extractor parts. One of the two features extractor parts is the hybrid feature extractor (HFE) part and another is the convolutional neural network VGG19 based CNN feature extractor part. The HFE portion combines three feature extraction approaches into a single fused feature vector: Histogram-Oriented Gradient (HOG), Local Binary Pattern (LBP), and Speed Up Robust Feature (SURF). The $\mathrm{CNN}$ method also is used to extract additional features from test and training datasets. This two-feature vector is fused to design the classification model. This classifier performs the classification of dermoscopy images whether it is melanoma or non-melanoma skin cancer. The proposed methodology is performed on two ordinary datasets and achieved the accuracy $99.85 \%$, sensitivity $91.65 \%$, and specificity $95.70 \%$, which makes it more successful than previous machine learning algorithms.
\end{abstract}

\section{Keywords}

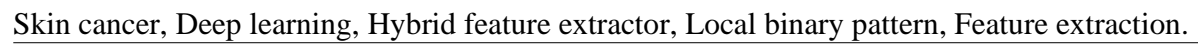

\section{Introduction}

Human skin is the largest organ that acts as the cover of the body[1]. Skin cancer appears when a cell of the skin grows abnormally, such as a hard red nodule or a scaly growth that produces a crust or a sore that does not heal[2]. So, lighter skin, sunburns or family history can increase the risk[3]. UV lights from the sun can damage the unprotected skin's DNA and alter the DNA. Thus, uncontrolled cell growth leads to cancer[4]. Squamous cell carcinoma, melanoma and basal cell carcinoma mutations may begin to alter the derma, resulting in skin cancer. Skin cancer shows such an alarming rate that according to WHO, currently more than 2 million non-melanoma skin cancers and around 130 thousand people are affected

\footnotetext{
${ }^{1}$ Department of Computer Science and Engineering, Mawlana Bhashani Science and Technology University, Tangail, Bangladesh.

${ }^{2}$ Department of CSE, Dhaka International University, Dhaka-1205, Bangladesh.

${ }^{3}$ Future Technology Research Center, College of Future, National Yunlin University of Science and Technology, 123 University Road, Section 3, Douliou, Yunlin 64002, Taiwan

4 Department of Computer Science, Rutgers University, Camden, NJ, 08102, USA

5 Center for Computational and Integrative Biology, Rutgers University, Camden, NJ, 08102, USA

${ }^{6}$ School of creative technologies University of Bolton United Kingdom

7 Department of Computing, Macquarie University, Sydney 2109, AU

${ }^{8}$ BioMedical Machine Learning Lab, The Graduate School of Biomedical Engineering, UNSW Sydney, Sydney, NSW, 2052,, Australia

${ }^{9}$ UNSW Data Science Hub, The University of New South Wales (UNSW Sydney), Sydney, NSW, 2052, Australia

${ }^{10}$ Health Data Analytics Program, AI-enabled Processes (AIP) Research Centre, Macquarie University, Sydney, 2109, Australia

* To whom correspondence should be addressed. E-mail: S.S.B (shamshirbands@yuntech.edu.tw)
} 
every year around the world $[5,6]$. Out of 5 stages, for melanoma stages $0,1,2$ is $98.4 \%$ survival rate. 63.6\% and 22.4\% for stages 3 and 4 respectively[7]. The American Cancer Society estimates about 7,180 from 106,110 patients is being dying in 2021 due to Melanoma[8]. If melanoma cancer spreads, it will be deadly, but in early stages it is curable. If we can detect melanoma skin cancer quickly, we can reduce human molarity rate with some initial treatment. Currently detection and level-based classification are mostly done by doctors manually, which may cause human error. Machine learning approaches can help to improve work speed and accuracy[9]. So, if we can detect cause as early as possible, death rate can be reduced and patients will be cured early.

Proposed system worked with a machine learning based system to classify melanoma and nonmelanoma skin cancer from dermatology images. In this system, input dataset is RGB image. First of all the proposed system truncates the selected Region of Interest (ROI) to erase the noisy and undesired parts and after that convert RGB to Gray scale image. Furthermore, the paper consists of two feature extractor parts. One of the two features extractor parts is the HFE part and the other is CNN based feature extractor part. The HFE part is used three feature extraction techniques such as HOG, LBP, and SURF which provide one fused feature vector. To extract features, the CNN approach is utilized, which results in another feature vector being discovered. This two-feature vector is mixed to design the classification model for training. Input dermatology images is speckle attacked and low-quality. An apostrophic diffusion filtering method is used to remove multiplicative noise present from the train and test images. It can efficiently overcome the image quality constraint in this way. The test images are then subjected to the feature extraction approach. Finally, the classifier determines whether or not dermatology images are melanoma or non-melanoma.

Melanoma skin cancer is on the rise right now as a result of a lack of early detection. Many people die of this cancer every year and a lot of human organs are damaged by this cancer. The proposed methodology has established a system that detect melanoma skin cancer very easily and enhance the survival rate of people. The key contribution of this research is:

Image Preprocessing: The input dermatology image is resized $(224 \times 224)$ by using MATLAB tools. The system used revised anisotropic diffusion filtering techniques after scaling to eliminate cumulative random noise in the input image.

Lesion Segmentation: The paper has used a threshold segmentation method to segment the background and object. For identify skin cancer region, first bounding box (FBB) method has applied.

Feature Extractor: For feature extraction the system consists of two parts. First part provides a combined feature vector which is a mixture of extraction of three features techniques such as HOG, LBP and SURF. Second part provides a feature vector by using VGG19 based CNN feature extraction techniques. This two-feature vector is fused in a form to classify. The proposed system has achieved a better accuracy.

\section{Related Work}

The medical and biological datasets are increasing rapidly. To analyse such big and complex data, artificial intelligence and machine learning algorithms become most popular [10-16]. Therefore, it is important to implement novel techniques to uncover the medical and biological patterns. In particular Machine learning and deep learning techniques have been widely used to analyse imaging data $[15,17$ 24]. Skin cancer is one of the most challenging medical; many researchers have used several methodology or techniques in melanoma skin cancer detection including dermatology image of skin.

For example, the author [25-27] introduced a novel system to detect melanoma skin cancer. The system has preprocessed skin lesion input image to get the high-quality image. The thresholding and edge detection techniques is used for segmentation. Then the system extract features from segmented image by using geometry-based features and $\mathrm{ABCD}$ (Asymmetry, Border, Color, and Diameter) features. These extracted features classified the image as ordinary skin and melanoma skin cancer. The research didn't clarify the accuracy in their proposed novel system. This study [28] provided an approach to detect normal skin and abnormal skin. First of all, the system preprocessed the dermoscopy image and segmentation by using threshold value. The gray level co-occurrence matrix was utilized to extract features, and feature selection was done using principal component analysis approaches. For 
classification the research applied support vector machine and calculate total dermoscopy score to get good accuracy. In 2018, the research conducted by Yuexiang Li and Linlin Shen. For lesion region detection, dermoscopic feature extraction, and classification, the researcher offered two deep learning algorithms. For segmentation and classification, Fully Convolutional Residual Networks (FCRN) are utilized, and the Lesion Index Calculation Unit (LICU) approach refines the classification outcomes calculation distance heat-map. Finally, CNN is used for dermoscopic feature extraction. The highest given accuracy using their framework is $91.2 \%$ [29]. Vijayalakshmi M et al. (2019) assessed the problem using three phases like data collection and augmentation, model design and prediction. The author used CNN, SVM algorithms and augmented it with different image processing tools and got $85 \%$ accuracy[30]. A. Pramanik and R. Chakraborty (2021) analyzed current technology to detect skin cancer. In their research K-Means clustering is used for image segmentation, Wavelet Transform for feature extraction and ANN, Feed forward ANN, CNN, SVM, BPN for classification where SVM provides 87.58\% accuracy[31]. Skin cancer categorization was demonstrated by Esteva et al. using a pre-trained Inception V3 CNN model. The researchers analyzed 129,450 clinical skin cancer images and 3,374 dermoscopic images and reported classification accuracy $72 \%$ [32]. K. Jayapriya et al. (2020) suggested a deep convolutional neural network-based layout. On the ISBI challenge dataset, the researchers built a fully convolutional residual network (FCRN) for real skin coup segmentation and produced 50 layers for the classification of melanoma cancer, with the best classification result of 88.92 percent accuracy[33]. Using Google's V4 CNN model, Haenssle et al. employed deep convolutional neural networks to identify skin cancer from dermoscopy images in 2018. In this research authors showed 86.6\% specificity[34]. This research priorities on convolutional neural network. Dorj et al. proposed a hybrid model to classify skin lesion cancer using AlexNet convolutional neural network and ECOC SVM methods. Pre trained AlexNet used for extracting features and ECOC SVM is used as a classifier. The authors reported maximum value of average accuracy is $95.1 \%$ [35].

\section{Research Methodology}

Fig. 1, shows the general framework of our proposed methodology. This section also includes a unique algorithm for the whole classification technique. In the next subsections, the requirements for each step of the suggested scheme are presented.

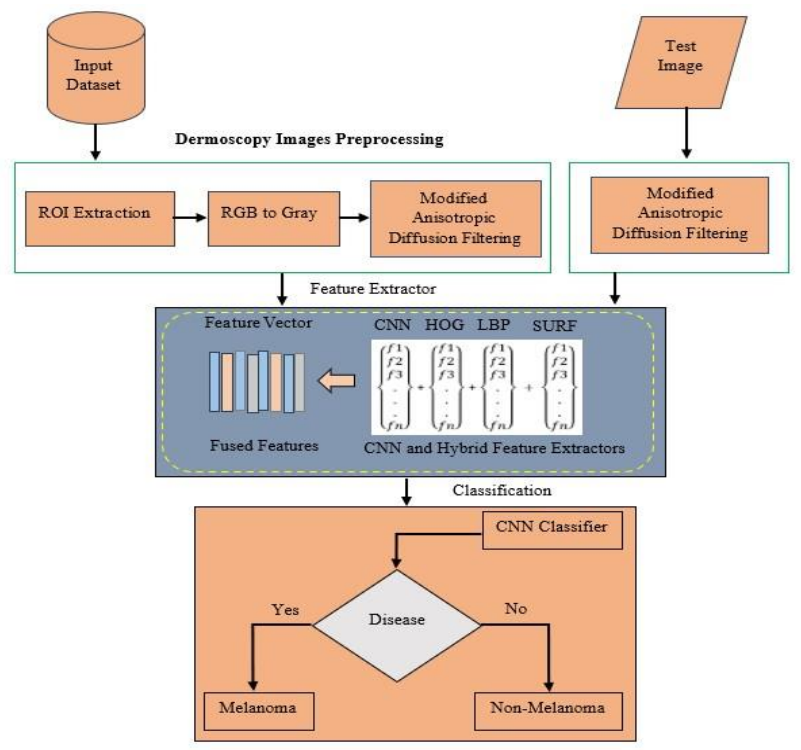

Fig. 1. Diagram of our proposed system. 


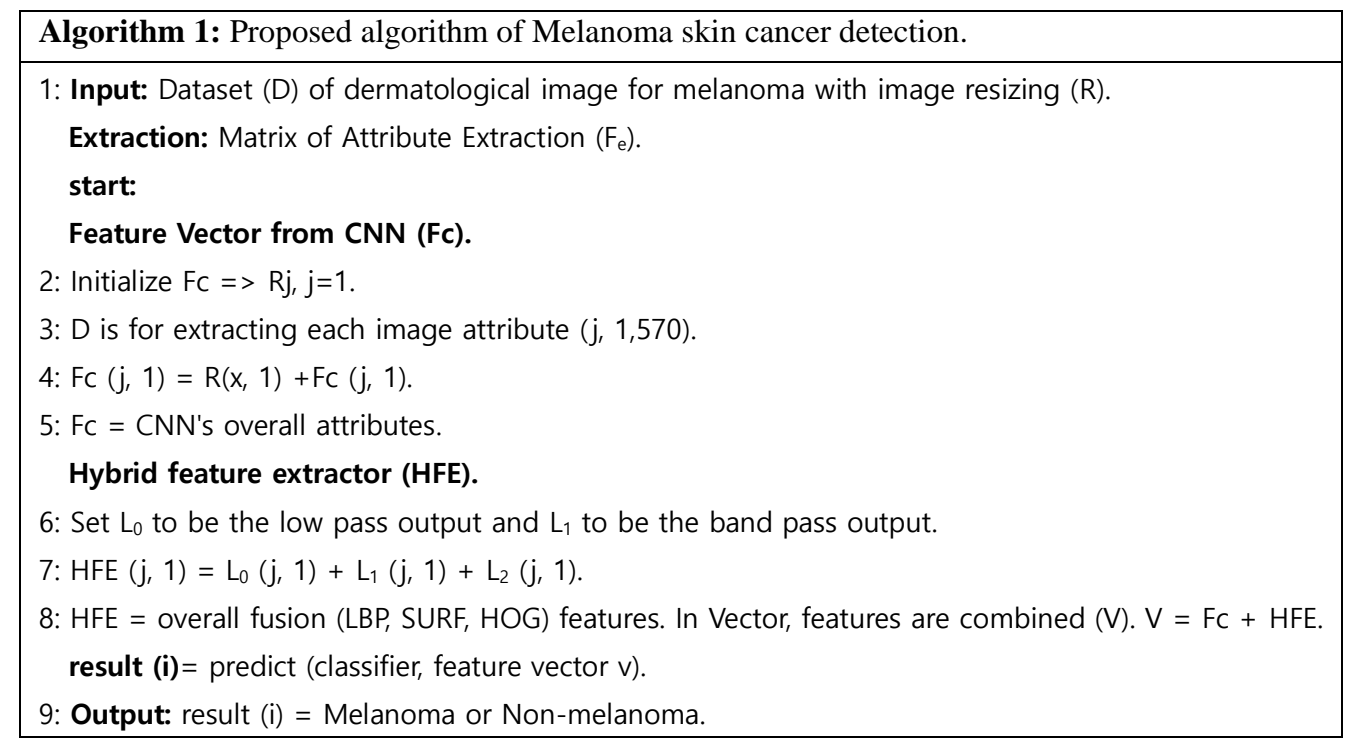

\subsection{Dataset}

By analyzing the performance of measurement matrices, the images are characterized as melanoma or non-melanoma. In this research, the paper used a standard dataset from Academic torrents and collected by HAM10000 (Human Against Machine with 10000 training images) dataset [36]. The dataset contains RGB 16,170 images in distinct types: melanoma and non-melanoma, which are publically available at (https://academictorrents.com and (https://isic-archive.com/). All of the skin cancer photographs have a resolution of $1980 \times 2022$. Two sections are included in the training and testing approach: one for melanoma and one for normal. The entire dataset is divided into two segments: 0.7 for training and 0.3 for testing. All of the training and testing took place on a 64-bit Windows 11 personal computer (PC). The PC has $8 \mathrm{~GB}$ of RAM and a $2.80 \mathrm{GHz}$ Intel 11th Gen Core i7 processor. The entire experiment was carried out in MATLAB 2019b. Fig. 2, Shows some samples image of our taken dataset.

\subsection{Data Preprocessing}
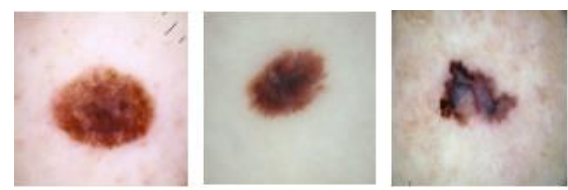

Data preprocessing is the most essential task for analysis before applying any feature extractor and classification methods [37-39]. For the Medical images preprocessing part is inevitable. Each image has a group of pixels that contains noise and imperfection. Some procedures are used to remove redundant pixels and distortion pixels from images in order to produce correct outcomes. After performing the ROI technique, the proposed system converts the RGB image to Grayscale. In order to remove irrelevant text and machine annotations from training and test images, the area of interest (ROI) is extracted[40]. This approach suppresses the number of unnecessary noise and distortion. Fig. 3 demonstrates the procedure of image data preprocessing. For any supervised learning for training phase, it is necessary to collect a huge volume of labeled data. In most applications, inadequate training data might lead to an overfitting issue $[21,41]$. By erasing the overfitting status, the data augmentation approach is able to overcome this barrier. The best fit in our model is a machine learning based on CNN model, which overcomes the limitation of the shortage of labeled images. Some of the augmentation techniques include translation, 
resizing, slicing, magnification, rotation, reversing, and brightness adjustment that may be used to change the size and appearance of a lesion in a dermatological image.

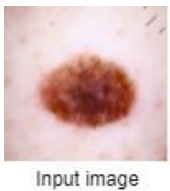

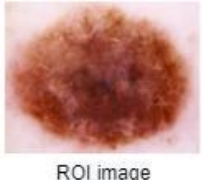

ROI image

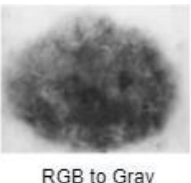

RGB to Gray

Fig. 3. Image data processing.

\subsection{Modified Anisotropic Diffusion Filtering}

The objective of proposed Modified Anisotropic Diffusion Filtering is preserving in detail information while speckles are being reduced. The suggested approach uses covariance and kurtosis measurements of noise to maintain the critical edge information. This speckle reduction technique is continued until the image's noise component reaches to Gaussian value. If the distortion is Gaussian, the skewness value must be 0 . Equation (1) represents the noise component. The loop will remain until the kurtosis of noise part is less than the measurement. This measurement can be defined by equation (3). When the relationship with both image class and disturbance class is the smallest, the iteration will end. In below equations (1) to (7), I and $I_{0}$ represent actual and noisy image, $\mu$ is used to represent the mean of noise intensity $\mathrm{G}$. The kurtosis value $\mathrm{k}$ is determined by equation (4). Equation (6) derives the image intensity correlation, whereas Equation (7) generates the noise intensity correlation. When I and G have the lowest amount of deviation, the recommended filtering will produce reliable results.

$$
\begin{gathered}
n=\frac{I-G}{\sqrt{G}} \\
I_{0}=I_{n} \\
\mu=\frac{\sum_{i=1}^{N} G_{i}}{N} \\
k=\frac{\frac{1}{N} \sum_{i=0}^{N}(G-\mu)^{4}}{\left[\frac{1}{N} \sum_{i=0}^{N}(G-\mu)^{2}\right]^{2}}-3 \\
a b s(n-k) \leq 0.001 \\
\rho_{I}=\frac{\sum_{i=0}^{M-1} \sum_{j=0}^{N-1} i . j . p_{I}(i, j)-\mu I_{x} \mu I_{y}}{\frac{\sum_{i=1}^{N}\left(I_{i x}-\mu_{I x}\right)\left(I_{i y}-\mu_{I y}\right)}{N}} \\
\rho_{G}=\frac{\sum_{i=0}^{M-1} \sum_{j=0}^{N-1} i . j . p_{G}(i, j)-\mu G_{x} \mu G_{y}}{\frac{\sum_{i=1}^{N}\left(G_{i x}-\mu_{I x}\right)\left(G_{i y}-\mu_{I y}\right)}{N}}
\end{gathered}
$$

Fig 4 depicts a visual comparison of speckle-reducing anisotropic diffusion, Bayesian NLM-demised, Memory based Speckle Statistics, and our recommended Modified Anisotropic Diffusion.

\subsection{Feature Extraction}

This section demonstrates applied different feature extractor techniques and their feature extraction process. In this research, two image extractors were used: HFE and a CNN-based feature extractor. HOG, LBP, and SURF are three feature extraction techniques included in the HFE extractor. To diagnose illnesses from skin dermatological images, the feature extractor techniques such as LBP, HOG, SURF, and neural network-based features extracts hybrid features. Numerous machine learning and computer 
vision applications use data fusion [19, 42-44]. The challenge of combining many feature vectors, known as features fusion, is critical. The technique proposed is based on entropy-based feature fusion. The vector that has been fused $(1 \times 1280)$. The entropy is applied to the features vector for the selection of optimal attributes based on the score. Equation (8) and (9) explain how the feature selection method works mathematically. From a total of 7948 characteristics, entropy was utilized to choose 1280 score-based characteristics. In Equations (8) and (9), indicates entropy and denotes features probability. In order to classify melanoma skin cancer images, the final attributes are sent to the classifiers.

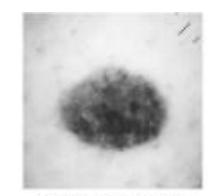

Original Image

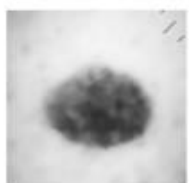

Spackle Reducing Anisotropic Diffusion

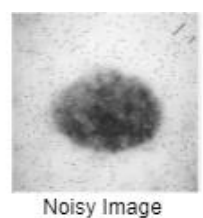

Noisy Image

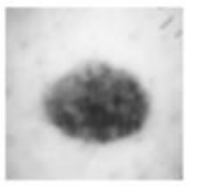

Denoised by Bayesian NLM

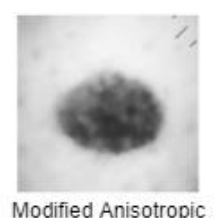
Diffusion

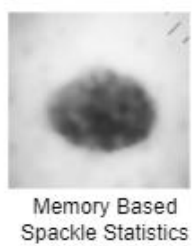

Fig. 4. Comparison among different Anisotropic Diffusion techniques.

$$
\begin{aligned}
B_{H e} & =-N H e_{b} \sum_{i=1}^{n} p(f) \\
F_{\text {select }} & =B_{H e}\left(\max \left(f_{i}, 1280\right)\right)
\end{aligned}
$$

Figure 5 depicts the segmentation and selection procedure. The proposed method has been tested on a fused features vector that includes both hybrid and deep learning features.

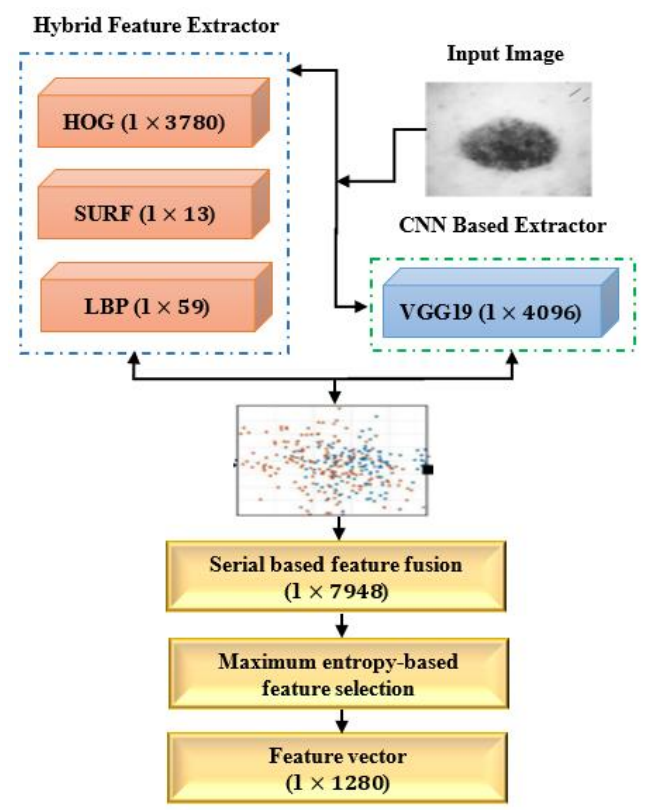

Fig. 5. Combination of feature fusion extractor. 


\subsection{Hybrid Feature Extractor}

Three extracting features strategies are included in the HFE extractor: local binary pattern (LBP), histogram-oriented gradient (HOG), and speed up robust feature (SURF), all of which result in a single fused feature vector. In the proposed system, hybrid features extractor i.e., LBP (12), SURF (11) and HOG (10) features are extracted. A fused features vector is utilized to evaluate the suggested approach. HOG properties are extracted from the images at all grid dense regions and are often utilized for object detection. HOG attributes $(1 \times 3780)$, LBP attributes $(1 \times 59)$, and SURF attributes $(1 \times 13)$ may all be used to define the form and appearance of skin cancer.

$$
\begin{aligned}
& f_{H O G 1 x n}=\left\{H O G_{1 x 1}, H O G_{1 x 2}, H O G_{1 x 3} \ldots H O G_{1 x n}\right\} \\
& f_{S U R F 1 x m}=\left\{S U R G_{1 x 1}, S U R F_{1 x 2}, S U R F_{1 x 3} \ldots S U R F_{1 x m}\right\} \\
& f_{L B P 1 x p}=\left\{L B P_{1 x 1}, L B P_{1 x 2}, L B P_{1 x 3} \ldots L B P_{1 x p}\right\}
\end{aligned}
$$

Furthermore, the extracted features are combined into one vector.

$$
\text { Fused (features vector })_{1 x q}=\sum_{i=1}^{3}\left\{f_{\text {HOG } 1 x n}, f_{\text {SURF } 1 x m}, f_{L B P 1 x p}\right\}
$$

\subsection{Histogram Oriented Gradient Features}

This research used a feature extraction algorithm known as HOG. Firstly, the input image is converted into gray scale image after that image transformed into gradient image for better edge detection. The gradients or edges orientation histogram is obtained in each cell unit, divided into smaller cells, and then these histograms are combined to give a HOG description[45, 46]. Fig. 6 depicts the HOG feature extraction algorithm's fundamental flow.

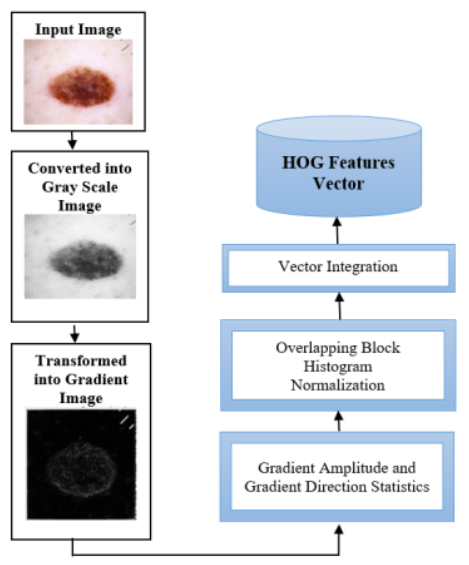

Fig. 6. The HOG extracting features algorithm's basic process.

To extract the feature in HOG, we should first create a gradient on both the $\mathrm{x}$ and $\mathrm{y}$ axis. $\mathrm{X}$ and $\mathrm{y}$ direction slopes can be determined fast since the horizontal direction's pattern is $\mathrm{K}=[-1,0,1]$, and its inversion may be used to filter an image. The following is the indication:

$$
\begin{aligned}
& g_{a}=I(x+1, y)-I(x-1, y) \\
& g_{y}=I(x, y+1)-I(x, y-1)
\end{aligned}
$$

The pixel value of $(x, y)$ is indicated by $I$, and the direction slope of $x$ is represented by $g_{x}$, while the orientation slope of $y$ is indicated by $g_{y}(x, y)$. The slope magnitude of $(x, y)$ is represented by $g(x, y)$ and denoted by

$$
\Delta \mathrm{g}(\mathrm{x}, \mathrm{y})=\sqrt{ }\left(\mathrm{gx}^{2}+\mathrm{gy}^{2}\right)
$$


And $(\mathrm{x}, \mathrm{y})$ gradient 's direction $(\theta)$ is determined as follows:

$$
\theta=\arctan \left(g_{y} / g_{x}\right)
$$

\subsection{Local Binary Pattern (LBP) Features}

LBP provides texture analysis and local spatial statistics of ultrasound image[47]. A threshold value is used to level the contiguous pixels and it is represented by 0 and 1 . If each pixel value is larger than the center pixel value, each adjacent pixel gray value $(3 \times 3)$ is leveled as 1 , otherwise it is leveled as 0 . Thus, LBP represents a set of binary digits which are used to replace center pixel value after converting into decimal. Equation (18), (19) represent LBP segmentation from test image where $g(p)$ is gray level pixel for surrounding pixels (i, j) and $g(c)$ is complementary constant. For neighbor $(8, i)$, the total number of samples is 256 .

$$
\begin{gathered}
l=\sum_{P=0}^{P-1} S\left(g_{p}-g_{c}\right) 2^{P} \\
S= \begin{cases}1 ; & \text { if } l(i, j)>0 \\
0 ; & \text { otherwise }\end{cases}
\end{gathered}
$$

\subsection{Speed Up Robust Feature (SURF) Features}

SURF is a similarity invariant representation and comparison algorithm. Its robust feature extractor technique is used in nearest neighbor matching[48]. During augmentation it can extract features. As a scaling and rotation variant algorithm, SURF provides fast operator computation using box filtering[49]. The two functions of SURF are feature extraction and feature description. The features extraction in SURF is done with Hessian matrix-based interest point approximation. The SURF descriptor provides unique information of features generated by surrounding area of an interest point. It operates by indicating the distinctive orientation of an interesting point using Haar wavelet responses. Before calculating descriptor, interest areas of neighbor interest point are rotated to its selected orientation. The Hessian matrix $\mathrm{H}(\mathrm{x}, \mathrm{y})$ at scaling is given by formula (20) for a given location $\mathrm{X}=(\mathrm{x}, \mathrm{y})$. Equation (21) represents the wavelet response in $\mathrm{x}$ and $\mathrm{y}$ direction is noted by $\mathrm{dx}$ and dy direction. A vector $\mathrm{V}$ is computed for each sub region.

$$
\begin{aligned}
& H(\dot{x}, \sigma)\left[\begin{array}{ll}
L_{x x}(x, \sigma) & L_{x y}(x, \sigma) \\
L_{x y}(x, \sigma) & L_{y y}(x, \sigma)
\end{array}\right] \\
& V=\left\{\sum d x, \sum d y, \sum|d x|, \sum|d y|\right\}
\end{aligned}
$$

\subsection{CNN Based Feature Extraction and Classification}

CNN based feature extraction techniques are most popular process in medical image processing [50]. In proposed research a pre-trained CNN model and scratch model were applied to extract features. Comparatively CNN (VGG19) provides good performance than scratch model. Pre-trained VGG19 model was well predictive and more performable feature extractor technique for our dataset among VGGNet, VGG16, Scratch model, ResNet50 and AlexNet.

19-layers are used to develop the network model using VGGNet techniques. Performance of given dataset using VGG19 is more accurate and reliable compared with others model. Fig. 7 shows how VGG19 was constructed using 16 convolution layers and three completely linked layers. The convolution component is separated into 5 successive max-pooling layers, with a nonlinear ReLU function acting as an activation function to ensure that each convolution layer's output is more accurate. Depth of the 5 consecutive layers is $64,128,256,512$ and 512 respectively. Each of the layer formatted with sub regions where pooling layer decrease the learnable parameter. Ending layer played a vital rule to get feature vector of proposed VGG19 model. Every fully attached layer besides the dropout layers was regularized with $\mathrm{L} 2$ to reduce overfitting problems during implementation of the fine tune model. Applying ReLU 
function on VGG19 based CNN model produce 4096 tuned features for further process. The features of VGG19 based CNN model and Hybrid feature extractor features are fused on a fused vector. The SoftMax feature aids in determining if the disease is Melanoma or Non-melanoma. The graphical illustration is shown in fig. 7.

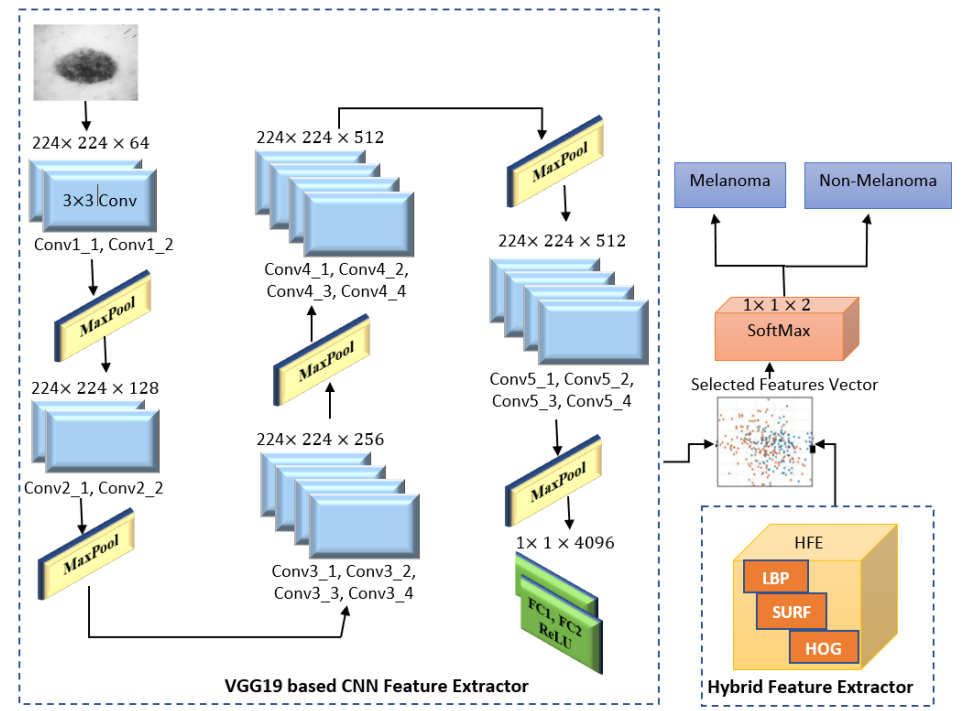

Fig. 7. CNN and HFE based proposed architecture.

\subsection{Finding a Cancer Region}

If the image is identified as a skin infection, the output section in this research is advised to determine the possibly damaged region. Depending upon the nature of the lesion, skin lesions appear in a range of forms and sizes. A circle is drawn around any spot that has been identified as a lesion.

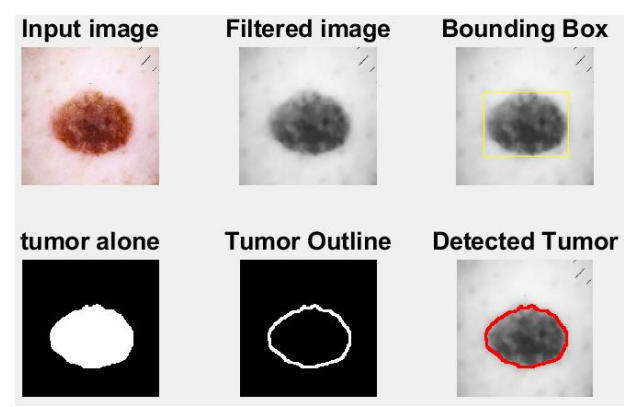

Fig. 8. Detect fracture region from skin cancer.

Fig. 8 shows the process of divulging tumor from skin lesion images. Dermoscopic skin lesion images are taken in training phase. Input images are transformed from RGB to gray scale prior to preprocessing. A modified anisotropic diffusion filtering method is used to the intended pictures to remove undesired noises. Filtered images provide an accurate region of tumor when fast bounding box is applied on it. Sometimes variant segmentation techniques are used to tumor detection which are not convenient and efficient due to deformation growth of lesion. Finding accurate region of skin lesion and symmetry of axis is time consuming and challenging. Researchers nowadays uses fast bounding box to detect lesion faster and robust. A fast bound box technique is fast and robust process of segmentation which overcome the above problem by locating an axis parallel box or bounding box around the skin lesion. This process is scored based on gray scale intensity analysis. Score function provides a linear search method for bounding boxes. FBB is an unsupervised and real time basis process where images fixation is not 
mandatory. Using this boundary box symmetry method tumor region can be detected alone finally which provides an outlier around the tumor.

\section{Experimental Setup}

The suggested system was evaluated using four performance criteria: accuracy, sensitivity, specificity, and precision. The parameters used to compute each measure are True Positive (TP), False Positive (FP), True Negative (TN), and False Negative (FN). In Table 1, confusion metrics are derived by calculating different values of TP, TN, FP and FN. Equation (22), (23), (24), and (25) represents the computing formula of four performance metrics.

$$
\begin{aligned}
& \text { Accuracy }(\mathrm{ACC})=\frac{\boldsymbol{T} \boldsymbol{P}+\boldsymbol{T N}}{\boldsymbol{T P + T N + F P + F \boldsymbol { N }}} \\
& \text { Sensitivity (SEN) }=\frac{\boldsymbol{T P}}{\boldsymbol{T P + F \boldsymbol { N }}} \\
& \text { Specificity (SPEC) }=\frac{\boldsymbol{T N}}{\boldsymbol{T N + \boldsymbol { F P }}} \\
& \text { Precision (PREC) }=\frac{\boldsymbol{T P}}{\boldsymbol{T P}+\boldsymbol{F P}}
\end{aligned}
$$

In Table 1, confusion metrics are derived by calculating different values of TP, TN, FP and FN. Equation (22), (23), (24), and (25) represents the computing formula of four performance metrics.

Table 1. Confusion Metrics of Dataset.

\begin{tabular}{lcc}
\hline True label & Melanoma & Non-melanoma \\
\hline Melanoma & TP (11076) & FN (120) \\
Non-melanoma & FP (94) & TN (4880) \\
\hline
\end{tabular}

\section{Results and Discussion}

Dermatology skin images with various speckles, noises, and resolutions were employed as test data for the proposed approach. Information reserve and noise reduction are required when working with relevant features. In the image preprocessing stage, the desired system employed modified anisotropic diffusion filtering. Three evaluation metrics are used to quantify performance in modified anisotropic diffusion filtering: Edge Preservation Factors (EPF), Minimum Square Error (MSE), and Signal to Noise Ratio (SNR). Higher SNR and EPF values indicate superior noise removal and edge detail preservation, respectively, when using the proposed filtering strategy. The minimum MSE value, and on the other hand, shows that there is less inconsistency between the input and filtering images. Table 2 represents the performance measurement using evaluation metrics. Although all known filters perform well in MSE, the proposed modified anisotropic filtering approach has a higher SNR and EPF.

Table 2. Anisotropic Diffusion-Based Filtering Algorithms to Assess Performance.

\begin{tabular}{cccc}
\hline \multirow{2}{*}{ Methods } & \multicolumn{3}{c}{ Evaluation Criteria } \\
\cline { 2 - 4 } & SNR & EPF & MSE \\
\hline SRAD & 31.3683 & 0.7218 & 0.6648 \\
OBLMN & 30.2361 & 0.7147 & 0.6750 \\
ADMSS & 33.0854 & 0.7298 & 0.6846 \\
Proposed MADF & 36.9687 & 0.9522 & 0.7389
\end{tabular}

CNN models like VGG-16, AlexNet, VGG-19, and ResNet50 are increasingly often used to feature extracted from training and testing data sets. VGG-19 outperforms other pre-trained algorithms in the proposed approach. All of these models provide the same result when given a similar training dataset. Table 3 represents performance of different CNN models for experimental data. However, 
proposed system also compares with some scratch models where performance is not satisfactory like fine-tuned models. CNN classifier is used to detect accuracy as classification scheme. Before classifying, $\mathrm{CNN}$ extracted features to train. Test characteristics were retrieved from a test image using multiple preprocessed models to evaluate the performance of $\mathrm{CNN}$ models.

Table 3. Performance Measurement of Different CNN Models.

\begin{tabular}{cccc}
\hline CNN Architecture & Accuracy & Specificity & Sensitivity \\
\hline Scratch model & 0.8637 & 0.8345 & 0.8192 \\
AlexNet & 0.8727 & 0.8528 & 0.8343 \\
ResNet50 & 0.9534 & 0.9345 & 0.9123 \\
VGG-16 & 0.9153 & 0.8732 & 0.8923 \\
VGG19 & 0.9849 & 0.9460 & 0.9165 \\
\hline
\end{tabular}

Table 3 shows that fine-tuned VGG19 pre-trained model provides better result in feature extraction using CNN. ResNet50 and VGG19 pre-trained models perform significantly better than VGG16 and AlexNet models. In term of accuracy VGG19 is selected for our test dataset though ResNet50 shows better sensitivity.

Table 4 depicts a comparison of several feature extraction methods. This study presents a fusion vector based on an HFF and CNN combination. For both the test and training samples, this fusion vector is used as the ultimate vector. Where CNN features are extracted from best fine-tuned VGG19. Hybrid feature extractor (HFF) is a semi fusion vector of Local Binary Pattern (LBP) Histogram Oriented Gradient (HOG), and Speed up Robust Feature (SURF). The individual performance values of different features extraction techniques are less satisfactory than fusion vector. In the final classification, the suggested fused feature performs well and classify accurately than using single approaches.

Table 4. Comparative Result of Feature Fusion.

\begin{tabular}{cccc}
\hline Proposed Methods & Accuracy & Specificity & Sensitivity \\
\hline CNN & 0.9473 & 0.8752 & 0.8673 \\
LBP & 0.9373 & 0.9273 & 0.9173 \\
SURF & 0.9773 & 0.9473 & 0.9373 \\
HOG & 0.9473 & 0.9373 & 0.9273 \\
HFF & 0.9773 & 0.9673 & 0.9473 \\
Our Proposed & 0.9949 & 0.9570 & 0.9165 \\
(CNN+HFF) & & & \\
\hline
\end{tabular}

Without preprocessing the performance result proposed fused features and CNN is not satisfactory. Table 5 shows accuracy, sensitivity and specificity result of noisy and speckle test dataset.

Table 5. Results of Test Data Segmentation without Pre-Processing.

\begin{tabular}{llll}
\hline Methods & Accuracy & Specificity & Sensitivity \\
\hline CNN only & 0.9273 & 0.8552 & 0.8773 \\
Proposed Fusion & 0.9675 & 0.9250 & 0.9100
\end{tabular}

Aside from CNN, several machine learning methods such as Decision Tree (DT), Random Forest (RM), Artificial Neural Network (ANN), and K closest Neighbor (KNN) have been used to produce the final classification. Proposed fused vector features are fed to classification methods to find better classifier. Finally, as demonstrated in Table 6, CNN shows better performance as a classifier. The proposed system used a starburst pattern and poor dermatological images during the testing phase. A modified apostrophic diffusion filtering approach is used to eliminate multiplicative noise present in the test image. It is capable of efficiently overcoming the challenges of a noisy image. As a result, the preprocessing approach for the input text image is more successful in reliably extracting features using the suggested fused vector. 
Table 6. Comparative Results of Different Classifier.

\begin{tabular}{llll}
\hline $\begin{array}{l}\text { Classifier } \\
\text { Models }\end{array}$ & Accuracy & Specificity & Sensitivity \\
\hline DT & 0.8128 & 0.8023 & 0.7978 \\
RF & 0.8326 & 0.8293 & 0.8027 \\
ANN & 0.8756 & 0.8634 & 0.8523 \\
KNN & 0.9123 & 0.8823 & 0.8732 \\
SVM & 0.9532 & 0.9423 & 0.9343 \\
CNN & 0.9985 & 0.9570 & 0.9165 \\
\hline
\end{tabular}

The bulk of study based on dermatological images has poor generalization accuracy. For generalization, the proposed system employed a standard dataset from an academic torrent. In generalization, we have used 16,170 images with two classes of 11170 images are melanoma and 5,000 images are non-melanoma. Table I displays the generalization findings' confusion metrics.

The cancer affected mole can come in many variant colors like brown, black and tan. The variety of mole in the same mole could be cancerous[20,51]. Finding the limitations of dermatological image was the first issue in this study. The area of interest (ROI) is retrieved from training images to eliminate extraneous text and machine classifications. Prior to that, the image was transformed from RGB to grayscale to eliminate the problem of multiple colors.

Detecting skin cancer using the same dataset or a related dataset is a tough issue. Preprocessing techniques, feature extraction approaches, and classification methods were all utilized by the researchers. It's now challenging to recommend a prospective strategy or combination of procedures for removing speckles from dermatological images that is more beneficial. In the phase of feature extraction and noise removal, the suggested approach presents a feasible technique. Table 7 shows the comparative analysis of relevant research. Table 5 shows that the suggested approach of feature fusion utilizing HFF and CNN (VGG19) performs greater accuracy than the CNN classifier. For dermatological datasets, CNN performs well as a binary classifier. To improve performance, modified anisotropic filtering of the input test image is essential. This preprocessing technique suppresses the limitation of test noisy image.

To obtain a more detailed experimental outcome, a k-cross validation procedure is used. The CNN system is built using a 5-fold classification technique after extracting features. This stage divides the feature vector into five sub folds at random. Four sub folds are chosen from the training dataset, whereas only one sub fold is chosen from the testing dataset. Table 8 shows the various results obtained utilizing the individual and combined methods.

Table 8. 5-Fold Cross Validation was used to evaluate overall classification accuracy.

\begin{tabular}{lllllll}
\hline Feature extraction techniques & Fold 1 & Fold 2 & Fold 3 & Fold 4 & Fold 5 & Mean Accuracy \\
\hline HFF & 0.8732 & 0.8789 & 0.8741 & 0.8675 & 0.8730 & 0.8734 \\
CNN & 0.9378 & 0.9367 & 0.9387 & 0.9367 & 0.9321 & 0.9364 \\
Proposed fusion (HFF+ CNN) & 0.9956 & 0.9987 & 0.9913 & 0.9997 & 0.9973 & 0.9949 \\
\hline
\end{tabular}

Table 8 represents the accuracy result of individual technique for each sub fold for LBP, SURF, HOG and VGG19 CNN model. Single feature extraction method provides less than $94 \%$ accuracy where proposed fused feature vector obtains $99.49 \%$ mean accuracy.

Table 7. Existing melanoma detection algorithms are compared.

\begin{tabular}{|c|c|c|c|}
\hline Authors & Dataset & Methodology & Accuracy \\
\hline Yuexiang Li et al. [14] & 2000 images & $\begin{array}{l}\text { Fully convolutional residual networks }(\text { FCRN })+\text { Lesion } \\
\text { index calculation unit }(\mathrm{LICU})+\mathrm{CNN}\end{array}$ & $91.2 \%$ \\
\hline $\begin{array}{l}\text { Vijayalakshmi M et al. } \\
\text { [15] }\end{array}$ & $\begin{array}{l}1000-1500 \\
\text { images }\end{array}$ & $\mathrm{CNN}+\mathrm{SVM}$ & $85 \%$ \\
\hline $\begin{array}{l}\text { A. Pramanik and R. } \\
\text { Chakraborty }[16]\end{array}$ & 100 images & $\mathrm{ANN}+\mathrm{SVM}+\mathrm{CNN}+\mathrm{BPN}$ & $87.58 \%$ \\
\hline
\end{tabular}




\begin{tabular}{|c|c|c|c|}
\hline $\begin{array}{l}\text { Andre Esteva et al. } \\
{[17]}\end{array}$ & 129,450 images & $\mathrm{CNN}$ & $72.1 \%$ \\
\hline K. Jayapriya et al. [18] & & $\mathrm{CNN}+\mathrm{FCRN}$ & $88.92 \%$ \\
\hline Haenssle et al. [19] & 300 images & Deep CNN+ Google Inception V4 & $86.6 \%$ \\
\hline Dorj et al. [20] & 3753images & $\mathrm{ECOC} \mathrm{SVM}+\mathrm{CNN}$ & $95.1 \%$ \\
\hline Proposed Method & $\begin{array}{l}16170 \\
\text { dermatology } \\
\text { images }\end{array}$ & $\mathrm{MADF}+\mathrm{HFF}(\mathrm{HOG}+\mathrm{LBP}+\mathrm{SURF})+\mathrm{CNN}$ & $\begin{array}{l}\text { Accuracy= } \\
99.85 \%\end{array}$ \\
\hline
\end{tabular}

The proposed deep learning model has a great potential to be used on healthcare imaging data analysis including CXR images, brain imaging, etc. Using a deep neural network to detect and classify skin cancer is a tough challenge.

\section{Conclusion}

Today, it is important to use innovative techniques to identify medical biomarkers and classify disease including cancer [50-54]. The proposed system in this study provides an exploratory analysis through hybrid feature extractor and convolutional neural network to gain more features information and thus achieve a promising accurate result. A modified anisotropic filtering technique is used in dermatology test image to diverge speckle from noisy images. The proposed fused vector with CNN and HFF has proven $99.49 \%$ accuracy. The best performing CNN classifier used to detect whether it's melanoma or non-melanoma skin cancer and also established $99.85 \%$ accuracy. In future this work will be extended using more promising machine learning algorithm and design a system for remotely checkup from home.

\section{Acknowledgements}

Not applicable.

\section{Author's Contributions}

MR and SSB designed the study. MR, MK, NA, SIK wrote the manuscript; MR, MK, NA, SIK collected data. SSB, AR, AB, and HAR edited the manuscript; MR, MK, NA, carried out the analyses. MR, MK generated all figures and tables. HAR and AB were not involved in any analysis. HAR, AB, ID and SSB do not have any responsibility against scripts, analyses and figures. All authors have read and approved the final version of the paper.

\section{Funding}

Not applicable

\section{Competing Interests}

The authors declare that they have no competing interests.

\section{References}

1. Saba, T., Computer vision for microscopic skin cancer diagnosis using handcrafted and non-handcrafted features. Microscopy Research and Technique, 2021. 84(6): p. 1272-1283.

2. Habeshian, K.A. and B.A. Cohen, Nodules and Tumors. Pediatric Dermatology EBook, 2021: p. 133.

3. Wu, Y.P., et al., A pilot study of a telehealth family-focused melanoma preventive 
intervention for children with a family history of melanoma. Psycho-Oncology, 2020. 29(1): p. 148-155.

4. Wirunchit, S., et al. The study of UV protection materials. in AIP Conference Proceedings. 2018. AIP Publishing LLC.

5. Lomas, A., J. Leonardi-Bee, and F. Bath-Hextall, A systematic review of worldwide incidence of nonmelanoma skin cancer. British Journal of Dermatology, 2012. 166(5): p. 1069-1080.

6. Nestor, M.S., et al., ConSENSUS Guidelines on the use of superficial radiation therapy for treating nonmelanoma skin cancers and keloids. The Journal of clinical and aesthetic dermatology, 2019. 12(2): p. 12.

7. Miller, R., et al., Epidemiology and survival outcomes in stages // and III cutaneous melanoma: A systematic review. Melanoma management, 2020. 7(1): p. МMT39.

8. Siegel, R.L., K.D. Miller, and A. Jemal, Cancer statistics, 2020. CA: a cancer journal for clinicians, 2020. 70(1): p. 7-30.

9. Kanani, P. and M. Padole, Deep learning to detect skin cancer using google colab. International Journal of Engineering and Advanced Technology Regular Issue, 2019. 8(6): p. 2176-2183.

10. Dashti, H., Det al., Integrative analysis of mutated genes and mutational processes reveals seven colorectal cancer subtypes. bioRxiv, 2020. 2020.

11. Javanmard, R., Jet al., Proposed a new method for rules extraction using artificial neural network and artificial immune system in cancer diagnosis. Journal of Bionanoscience, 2013. 7(6): p. 665-672.

12. Mahmoudi, M.R., Akbarzadeh, H., Parvin, H., Nejatian, S., Rezaie, V., Consensus function based on cluster-wise two level clustering. Artificial Intelligence Review, 2021. 54(1): p. 639-665.

13. Niu, H., Khozouie, N., Parvin, H., Beheshti, A., \& Mahmoudi, M. R., An ensemble of locally reliable cluster solutions. Applied Sciences, 2020. 10(5): p. 1891.

14. Rajaei, P., Jahanian, K. H., Beheshti, A., Band, S. S., Dehzangi, A., VIRMOTIF: $A$ user-friendly tool for viral sequence analysis. Genes, 2021. 12(2): p. 186.

15. Shamshirband, S., Fathi, M., Dehzangi, A., Chronopoulos, A. T., A review on deep learning approaches in healthcare systems: Taxonomies, challenges, and open issues. Journal of Biomedical Informatics, 2021. 113: p. 103627.

16. Ghareyazi, A., Mohseni, A., Dashti, H., Beheshti, A., Dehzangi, A., Rabiee, H. R., Whole-genome analysis of de novo somatic point mutations reveals novel mutational biomarkers in pancreatic cancer. Cancers, 2021. 13(17): p. 4376. 
17. Ahmadinia, M., Energy-efficient and multi-stage clustering algorithm in wireless sensor networks using cellular learning automata. IETE Journal of Research, 2013. 59(6): p. 774-782.

18. Esmaeili, L., Hybrid recommender system for joining virtual communities. Research Journal of Applied Sciences, Engineering and Technology, 2012. 4(5): p. 500-509.

19. Niu, H., Deep feature learnt by conventional deep neural network. Computers \& Electrical Engineering, 2020. 84: p. 106656.

20. KHAN, A.H., et al., STATISTICAL FEATURE LEARNING THROUGH ENHANCED DELAUNAY CLUSTERING AND ENSEMBLE CLASSIFIERS FOR SKIN LESION SEGMENTATION AND CLASSIFICATION. Journal of Theoretical and Applied Information Technology, 2021. 99(5).

21. Khan, S.I., et al., Breast Invasive Ductal Carcinoma Classification Based on Deep Transfer Learning Models with Histopathology Images. Data Science and Data Analytics: Opportunities and Challenges, 2021: p. 249.

22. Alinejad-Rokny, H., Machine learning and data mining techniques for medical complex data analysis. Neurocomputing, 2018. 276(1).

23. Bayati, M., Rabiee, H. R., Mehrbod, M., Vafaee, F., Ebrahimi, D., Forrest, A. R., CANCERSIGN: a user-friendly and robust tool for identification and classification of mutational signatures and patterns in cancer genomes. Scientific reports, 2020. 10(1): p. 1-11.

24. Parvin, H., Behrouz Minaei, Hosein Alizadeh, and Akram Beigi, A novel classifier ensemble method based on class weightening in huge dataset, in In International Symposium on Neural Networks. 2011, Springer. p. 144-150.

25. Jain, S. and N. Pise, Computer aided melanoma skin cancer detection using image processing. Procedia Computer Science, 2015. 48: p. 735-740.

26. Patel, K., et al., A survey on artificial intelligence techniques for chronic diseases: open issues and challenges. Artificial Intelligence Review, 2021: p. 1-54.

27. Rejeesh, M., Interest point based face recognition using adaptive neuro fuzzy inference system. Multimedia Tools and Applications, 2019. 78(16): p. 2269122710.

28. Alquran, $\mathrm{H}$., et al. The melanoma skin cancer detection and classification using support vector machine. in 2017 IEEE Jordan Conference on Applied Electrical Engineering and Computing Technologies (AEECT). 2017. IEEE.

29. Li, Y. and L. Shen, Skin lesion analysis towards melanoma detection using deep learning network. Sensors, 2018. 18(2): p. 556. 
30. Vijayalakshmi, M., Melanoma skin cancer detection using image processing and machine learning. International Journal of Trend in Scientific Research and Development (IJTSRD), 2019. 3(4): p. 780-784.

31. Pramanik, A. and R. Chakraborty, A Deep Learning Prediction Model for Detection of Cancerous Lesions from Dermatoscopic Images, in Advanced Machine Learning Approaches in Cancer Prognosis. 2021, Springer. p. 395-423.

32. Esteva, A., et al., Dermatologist-level classification of skin cancer with deep neural networks. nature, 2017. 542(7639): p. 115-118.

33. Jayapriya, K. and I.J. Jacob, Hybrid fully convolutional networks-based skin lesion segmentation and melanoma detection using deep feature. International Journal of Imaging Systems and Technology, 2020. 30(2): p. 348-357.

34. Haenssle, H.A., et al., Man against machine: diagnostic performance of a deep learning convolutional neural network for dermoscopic melanoma recognition in comparison to 58 dermatologists. Annals of oncology, 2018. 29(8): p. 18361842.

35. Dorj, U.-O., et al., The skin cancer classification using deep convolutional neural network. Multimedia Tools and Applications, 2018. 77(8): p. 9909-9924.

36. Tschandl, P., C. Rosendahl, and H. Kittler, The HAM10000 dataset, a large collection of multi-source dermatoscopic images of common pigmented skin lesions. Scientific data, 2018. 5(1): p. 1-9.

37. Fan, C., et al., A Review on Data Preprocessing Techniques Toward Efficient and Reliable Knowledge Discovery From Building Operational Data. Front. Energy Res, 2021. 9: p. 652801.

38. Khan, M.S.I., et al., Water quality prediction and classification based on principal component regression and gradient boosting classifier approach. Journal of King Saud University-Computer and Information Sciences, 2021.

39. Akhtar, J., Non-Small Cell Lung Cancer Classification from Histopathological Images using Feature Fusion and Deep CNN.

40. Zhang, L. and H. Wu, Cosaliency Detection and Region-of-Interest Extraction via Manifold Ranking and MRF in Remote Sensing Images. IEEE Transactions on Geoscience and Remote Sensing, 2021.

41. Khan, S.I., et al., MultiNet: $A$ deep neural network approach for detecting breast cancer through multi-scale feature fusion. Journal of King Saud UniversityComputer and Information Sciences, 2021.

42. Nweke, H.F., et al., Data fusion and multiple classifier systems for human activity detection and health monitoring: Review and open research directions. 
Information Fusion, 2019. 46: p. 147-170.

43. Parvin, H., MirnabiBaboli, M., \& Alinejad-Rokny, H., Proposing a classifier ensemble framework based on classifier selection and decision tree. Engineering Applications of Artificial Intelligence, 2015: p. 34-42.

44. Bahrani, P., Minaei-Bidgoli, B., Parvin, H., Mirzarezaee, M., Keshavarz, A., User and item profile expansion for dealing with cold start problem. Journal of Intelligent \& Fuzzy Systems, 2020. 38(4): p. 4471-4483.

45. Hussein, I.J., et al., Fully-automatic identification of gynaecological abnormality using a new adaptive frequency filter and histogram of oriented gradients (HOG). Expert Systems, 2021: p. e12789.

46. Li, Y., et al. Research on Invasive Species Recognition Based on SVM+HOG. in 2021 2nd International Conference on Artificial Intelligence and Information Systems. 2021.

47. Kushwaha, R., G. Singal, and N. Nain, $A$ texture feature based approach for person verification using footprint bio-metric. Artificial Intelligence Review, 2021. 54(2): p. 1581-1611.

48. Hajano, S., B. Naz, and S. Talpur, Area and Feature Based Image Registration Using Template Matching and SURF Algorithm.

49. Liu, J., Q. Wei, and Y. Bai. Fast stitching of UAV images based on improved SURF algorithm. in 2021 IEEE 3rd International Conference on Civil Aviation Safety and Information Technology (ICCASIT). 2021. IEEE.

50. Barbhuiya, A.A., R.K. Karsh, and R. Jain, CNN based feature extraction and classification for sign language. Multimedia Tools and Applications, 2021. 80(2): p. 3051-3069.

51. Dhivyaa, C., et al., Skin lesion classification using decision trees and random forest algorithms. Journal of Ambient Intelligence and Humanized Computing, 2020: p. 1-13.

52. Alinejad-Rokny, H., Anwar, F., Waters, S. A., Davenport, M. P., \& Ebrahimi, D., Source of CPG depletion in the HIV-1 genome. Molecular biology and evolution, 2016. 33(12): p. 3205-3212.

53. Heidari, R., Akbariqomi, M., Asgari, Y., Ebrahimi, D., A systematic review of long non-coding RNAs with a potential role in Breast Cancer. Mutation Research/Reviews in Mutation Research, 2021. 787: p. 108375.

54. Hosseinpoor, et al., Proposing a novel community detection approach to identify cointeracting genomic regions. Mathematical Biosciences and Engineering, 2020. 17(3): p. 2193-2217. 